\title{
Surfactant and RDS: What's a Neonatologist to Do?
}

\author{
Donn SM* \\ Professor Emeritus of Pediatrics, Division of Neonatal-Perinatal Medicine, C.S. Mott Children's \\ Hospital, USA \\ Commentary \\ Volume 5 Issue 1 \\ Received Date: November 30, 2020 \\ Published Date: December 04, 2020
}

*Corresponding author: Steven M Donn, Professor Emeritus of Pediatrics, Division of

Neonatal-Perinatal Medicine, C.S. Mott Children's Hospital Michigan Medicine, Ann Arbor, MI 48103, USA, Tel: +17343520908;

Email: smdonnmd@med.umich.edu

\section{Commentary}

The creation of exogenous pulmonary surfactant has been one of, if not the most, significant breakthroughs in the field of neonatal medicine. The early clinical studies, utilizing either animal-derived surfactants or synthetic preparations, demonstrated an impressive improvement in the survival of preterm infants with respiratory distress syndrome, a disorder whose hallmark is surfactant deficiency. These trials involved the administration of surfactant directly into an indwelling endotracheal tube, necessitating endotracheal intubation, an invasive procedure in an unstable baby, often resulting in hypoxemia, desaturation, bradycardia, and increased intracranial pressure.

As neonatologists learned how to harness this new wonder drug, and as mechanical ventilation became safer and more sophisticated, surfactant continued to be instilled through the endotracheal tube during the 1980s and 1990s. Concurrently, two important changes also took place. First, following the NIH Consensus Statement on antenatal corticosteroid use, there was a marked increase in the treatment of pregnant women threatening preterm delivery. Second, the success of surfactant administration and mechanical ventilation changed the demographics of the neonatal intensive care unit (NICU), as smaller and even more premature infants began to survive.

In the new millennium, the pendulum began to move towards a greater use of non-invasive support of babies with RDS. Continuous positive airway pressure (CPAP) and its derivatives began to replace intubation and surfactant administration. Early clinical trials demonstrated comparable outcomes between non-invasive support and mechanical ventilation/surfactant, but the incidence of bronchopulmonary dysplasia (BPD), the leading long-term respiratory morbidity of RDS was unchanged. Moreover, among the most preterm infants, nearly half failed CPAP within the first week and required intubation and mechanical ventilation. Thus, neonatologists faced the dilemma: invasively intubate and give surfactant or avoid intubation but preclude surfactant therapy?

This dilemma led to attempts to modify the means of surfactant administration. One of the first novel approaches was dubbed INSURE, an acronym for intubate, surfactant, and extubate. The idea was to directly instill the surfactant in the typical clinical format but truncate the course of mechanical ventilation by quickly extubating to some form of CPAP. However, at the same time, concerns were raised about the need for pre-medicating with sedatives and/or analgesics for elective intubation, and these agents suppress spontaneous breathing and may prevent early extubation. A modification known as MIST (minimally invasive surfactant therapy) or LISA (less invasive surfactant administration) has subsequently been used. With this technique, laryngoscopy is performed and either a thin feeding tube or a vascular catheter is inserted into the airway, surfactant is squirted into the lungs, and the catheter is withdrawn. This technique requires a greater level of skill to accomplish, and in addition, the operator has minimal control of the airway in the event of airway obstruction, a not uncommon complication during surfactant administration.

Finally, a potential compromise is the administration of surfactant as an aerosol using CPAP. If successful, this would obviate the need for intubation and would assure surfactant repletion in the surfactant deficient newborn, provided there is active spontaneous breathing. Until recently, aerosolization has been difficult to accomplish. The energy required to aerosolize surfactant often led to its inactivation. Particle size had to be sufficient to penetrate the distal 


\section{Open Access Journal of Pulmonary \& Respiratory Sciences}

airways and alveoli, yet appropriate to avoid deposition in the upper airway, and there had to be delivery of a requisite concentration to be therapeutic. Delivered particles would then have to reaggregate and retain biologic function.

It now appears that both surfactant and device technology have evolved to the point where clinical trials have been underway to test this hypothesis. The first two trials (recently completed) used both a synthetic surfactant with a surfactant protein-B mimic, able to withstand the aerosolization process, and an animal-derived surfactant. A third trial has recently begun and is using an animal-derived surfactant with a novel delivery device.

If these trials are successful, the potential exists to redefine the standard of care for spontaneously breathing babies with RDS, avoiding both laryngoscopy and intubation, but still repleting surfactant deficiency, perhaps significantly reducing the need for mechanical ventilation and its attendant complications. It would be more than just building a better mousetrap. 